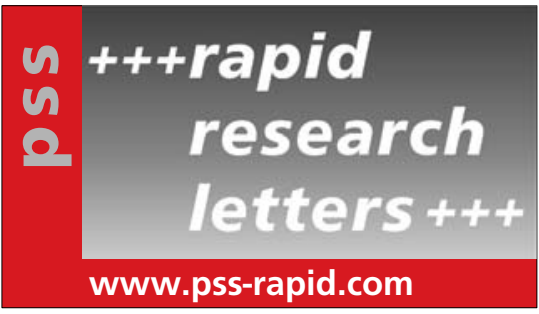

Review@RRL

\title{
Sublattice engineering and voltage control of magnetism in triangular single and bi-layer graphene quantum dots
}

\author{
A. D. Güçlü ${ }^{*}, 1$, P. Potasz ${ }^{2}$, and P. Hawrylak \\ ${ }^{1}$ Department of Physics, Izmir Institute of Technology, IZTECH, 35430 Izmir, Turkey \\ ${ }^{2}$ Department of Theoretical Physics, Wroclaw University of Technology, Wybrzeze Wyspianskiego 27, 50-370 Wroclaw, Poland \\ ${ }^{3}$ Physics Department, University of Ottawa, Ottawa, Canada K1N 6N5
}

Received 26 May 2015, revised 2 September 2015, accepted 2 September 2015

Published online 21 September 2015

Keywords two-dimensional materials, graphene, nanostructures, strongly correlated systems

${ }^{*}$ Corresponding author: e-mail devrimguclu@iyte.edu.tr, Phone: +90-232-7507713

When a Dirac electron is confined to a triangular graphene quantum dot with zigzag edges, its low-energy spectrum collapses to a shell of degenerate states at the Fermi level leading to a magnetized edge. The shell degeneracy and the total magnetization are proportional to the edge size and can be made macroscopic. In this review, we start with a general discussion of magnetic properties of graphene structures and its relation to broken sublattice symmetry. Then, we discuss single electronic properties of single and bilayer triangular graphene quantum dots, focusing on the nature of edge states. Finally, we investigate the role of electronic correlations in determining the nature of ground state and excitation spectra of triangular graphene quantum dots as a function of dot size and filling fraction of the shell of zero-energy states. The interactions are treated by a combination of tight-binding, Hartree-Fock and configuration interaction methods. We show that the spin polarization of the triangular graphene quantum dots can be controlled through gating, i.e., by adding or removing electrons. In bilayer graphene dots, the relative filling of edge states in each layer and the magnetization can be tuned down to single localized spin using an external vertical electrical field.
1 Magnetism and graphene As the electrons in carbon based materials tend to form covalent bonds, one does not expect to find significant magnetism in a carbon system. Indeed, pure graphene is not magnetic. However, as discussed here, there is a possibility to induce magnetism in graphene and graphene nanostructures [1-30] through electron-electron interactions and sublattice engineering which can potentially lead to a new class of devices for magnetic storage and spintronics.

Sublattice engineered magnetism in graphene rests on Lieb's theorem for the Hubbard model on bipartite lattice relating total spin to the broken sublattice symmetry [31]. Consider the general Hubbard Hamiltonian with a constant repulsive on-site interaction $U$

$$
H=\sum_{i, j, \sigma} t_{i j} c_{i \sigma}^{\dagger} c_{j \sigma}+U \sum_{i \sigma} n_{i \uparrow} n_{i \downarrow},
$$

where the elements $t_{i j}$ are assumed to be real and the lattice is bipartite, i.e., there are A and B types of sites (sublattice) such that $t_{i j}=0$ if $i$ and $j$ belong to the same sublattice. Then the theorem states that for a half-filled bipartite lattice with repulsive $U$, the ground state is unique and has a total spin $S=\left|N_{\mathrm{A}}-N_{\mathrm{B}}\right| / 2$ where $N_{\mathrm{A}}$ and $N_{\mathrm{B}}$ are number of sites in each sublattice.

Graphene is an ideal system that can be approximately described by a half-filled bipartite lattice: Each carbon 
atom provides one single $\mathrm{p}_{z}$ electron well decoupled from the rest of its electrons that ensure the $\mathrm{sp}^{2}$ bonding of the honeycomb lattice. Thus, in a pristine graphene structure, it should be sufficient to break the symmetry between the two sublattices consisting of two triangular lattices A and $\mathrm{B}$ in order to induce magnetization.

In principle, there are various ways of breaking the sublattice symmetry of graphene lattice by varying the shape and edge of graphene nanostructures or creating defects. For instance, one can create defects by removing Carbon atoms from the lattice [32] or add adatoms on top of Carbon atoms [33], which would induce a localized spin around the vacancy/impurity. The Lieb's theorem also predicts that if there are two vacancies or impurities in the lattice, they should couple to each other ferromagnetically or antiferromagnetically (depending on whether they lie on the same or opposite sublattices) over large distances [34]. This is illustrated in Fig. 1, where the magnetic coupling (more specifically the static spin-spin magnetic susceptibility) between two adatoms on opposite sublattices was calculated as a function of the distance between them (in units of second nearest neighbor distance $b$ ) by solving the Anderson model using a quantum Monte Carlo method. The calculations were performed for different inverse temperature values $\beta$. A comparison with the RudermanKittel-Kasuya-Yoshida (RKKY) model is also provided. These results show that the coupling between the impurities is indeed antiferromagnetic and strongly enhanced, becoming several orders of magnitude larger at longer distances as the temperature is lowered. A similar result was also obtained for adatom impurities sitting on the same sublattice, but coupled ferromagnetically [34].

Lieb's theorem for half-filled bipartite lattice has also important implications for graphene nanostructure with zigzag edges. Zigzag edges break the symmetry between

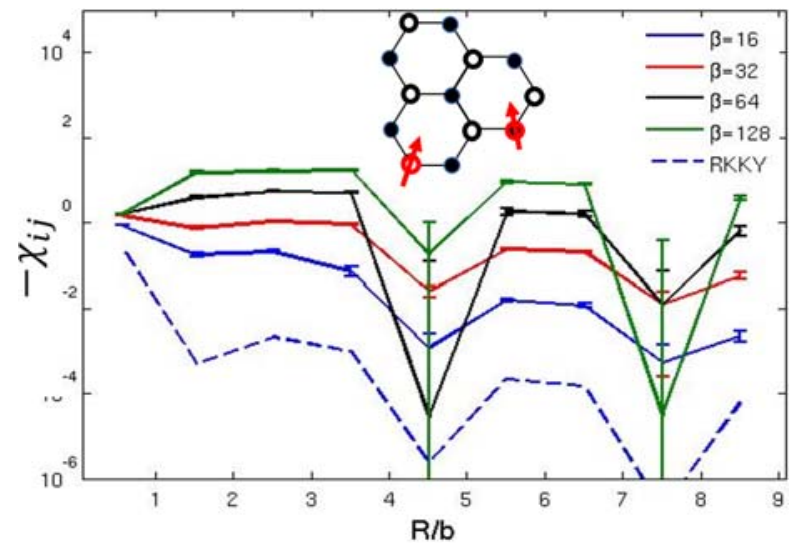

Figure 1 Static magnetic susceptibility between two magnetic adatom impurities along the zigzag direction as a function of distance (in units of second nearest neighbor distance $b$ ) for the $\mathrm{AB}$ configuration (impurities on opposite sublattices, shown in the inset) obtained by QMC calculations at different inverse temperatures $\beta$. The dashed lines are RKKY results. Reprinted from Ref. [34]. the two sublattices, hence one expects finite magnetism near those edges [35-38]. In particular, a class of graphene nanostructures called triangular shaped graphene quantum dots (TGQD) with zigzag edges [14-29] provides the highest ratio of $\left|N_{\mathrm{A}}-N_{\mathrm{B}}\right| /\left(N_{\mathrm{A}}+N_{\mathrm{B}}\right)$, yielding a maximum magnetization per atom. In TGQDs, the atoms sitting on all three edges of the triangle belong to the same sublattice and are expected to become spin polarized according to Lieb's theorem. This was explicitly shown by Ezawa [15] using Ising model, Fernandez-Rossier and Palacios [16] using meanfield Hubbard model, and by Wang et al. [17] using density functional calculations. Engineering those structures would open the door to a completely new class of magnetic devices for storage, sensors, and data processing. It is therefore important to understand the details of the physics of magnetism in those structures beyond Lieb's theorem and mean-field results, including size effects, gating, long-range, exchange and scattering terms in the graphene quantum dot Hamiltonian.

\section{Single particle properties of TGQDs}

2.1 One-band empirical tight-binding model The one-band empirical tight-binding model where the $\mathrm{sp}^{2}$ hybridized orbitals are neglected as discussed in this issue by Ozfidan et al., describes successfully the one-electron spectrum of bulk graphene [39]. For TGQDs, assuming passivation of edges with hydrogen, the tight-binding Hamiltonian in the second quantization form with only nearest neighbor hopping included can be written as

$$
H_{\mathrm{TB}}=t \sum_{\langle i, l\rangle, \sigma} c_{i \sigma}^{\dagger} c_{l \sigma},
$$

where $c_{i \sigma}^{\dagger}$ and $c_{i \sigma}$ are creation and annihilation operators for an electron on the lattice site $i$ with spin $\sigma$, and $\langle i, l\rangle$ indicates a summation over nearest neighbor sites. The hopping integrals between nearest $\mathrm{A}$ and $\mathrm{B}$ neighbor atoms corresponding to two sublattices are denoted by $t$.

A striking property of single particle spectrum of TGQDs with zigzag edges is the existence of a zero-energy shell at the Fermi level, with degeneracy related to the size of the triangle. TGQDs can be characterized by the number of atoms on one edge of the triangle (see the inset of Fig. 2(a) and (b)), $N_{\text {ed }}$, and the total number of atoms $N=N_{\text {ed }}^{2}+4 N_{\text {ed }}+1$ is expressed by the number of atoms at the edge. There are $N_{\mathrm{A}}$ and $N_{\mathrm{B}}$ atoms corresponding to sublattice $\mathrm{A}$ and $\mathrm{B}$. The difference between the number of atoms of types $\mathrm{A}$ and $\mathrm{B}$ is proportional to the number of atoms on one edge, $\left|N_{\mathrm{A}}-N_{\mathrm{B}}\right|=N_{\text {ed }}-1$.

Figure 2 shows the TB energy spectra of two TGQDs with different sizes. Figure 2(a) corresponds to the structure consisting of $N=78$ atoms, or $N_{\text {ed }}=7$, and Fig. 2(b) to the structure consisting of $N=97$ atoms or $N_{\text {ed }}=8$. From numerical diagonalization of the TB Hamiltonian we find $N_{\mathrm{deg}}=6$ and $N_{\mathrm{deg}}=7$ degenerate states at the Fermi level, respectively. The number of degenerate states $N_{\text {deg }}$ in these TGQDs is related to the number of edge atoms as $N_{\text {deg }}=N_{\text {ed }}-1=N_{\mathrm{A}}-N_{\mathrm{B}}$. In the next section we will show 
(a)

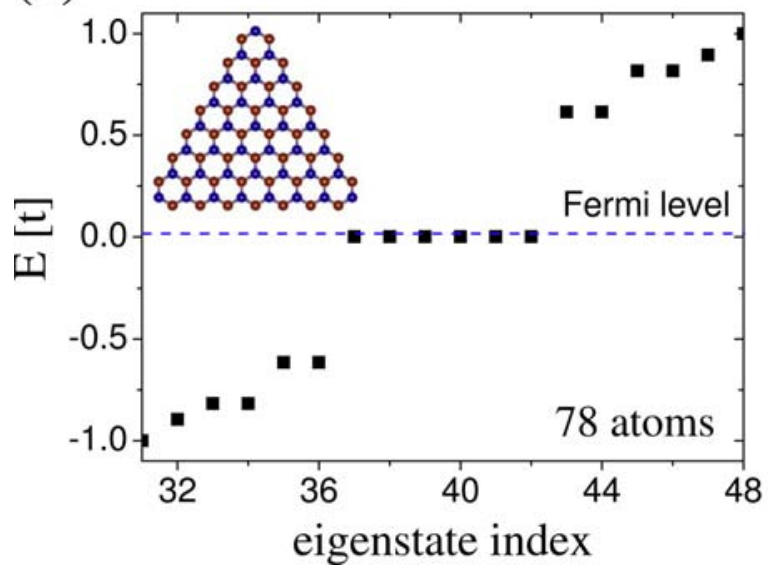

(b)

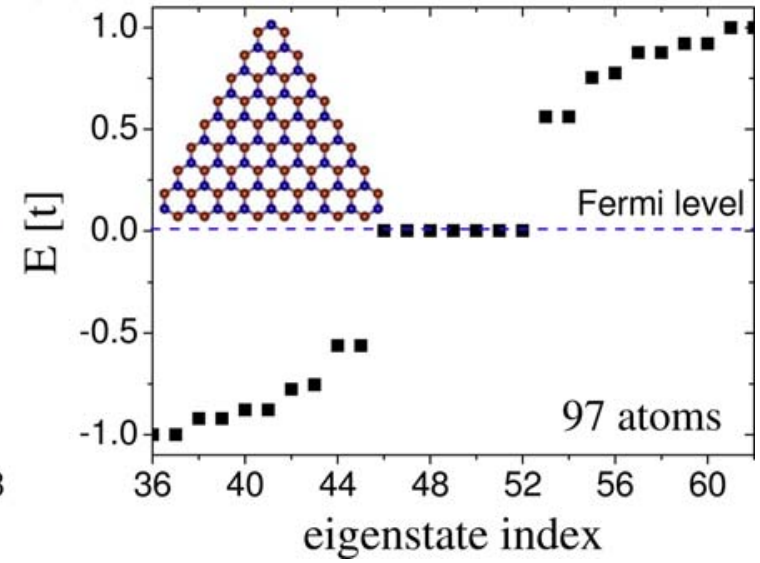

Figure 2 TB energy spectra of TGQDs consisting of (a) $N=78$ atoms, where the number of atoms on one edge of the triangle is given by $N_{\text {ed }}=7$ and the number of degenerate edge states at the Fermi level is $N_{\text {deg }}=6$, and (b) $N=97$ atoms where $N_{\text {ed }}=8$ and $N_{\text {deg }}=7$. Reprinted from [1].

that this is a general rule for all TGQDs; by increasing the size of triangles the degeneracy of the zero-energy shell increases and can be made macroscopic.

2.2 Analytical proof of zero-energy states We will now prove that the number of zero-energy states is related to the number of edge atoms through the relation $N_{\text {deg }}=N_{\text {ed }}-1=N_{\mathrm{A}}-N_{\mathrm{B}}$, and provide an analytical solution to the edge states [23].

For an arbitrary B-type site surrounded by three A-type sites, shown in Fig. 3(a), the wavefunction written in a basis of $\mathrm{p}_{z}$ orbitals $\varphi_{z}$ has the following form:

$$
\Psi=b_{i} \varphi_{z}^{i}+b_{j} \varphi_{z}^{j}+b_{k} \varphi_{z}^{k}+b_{l} \varphi_{z}^{l},
$$

(a)
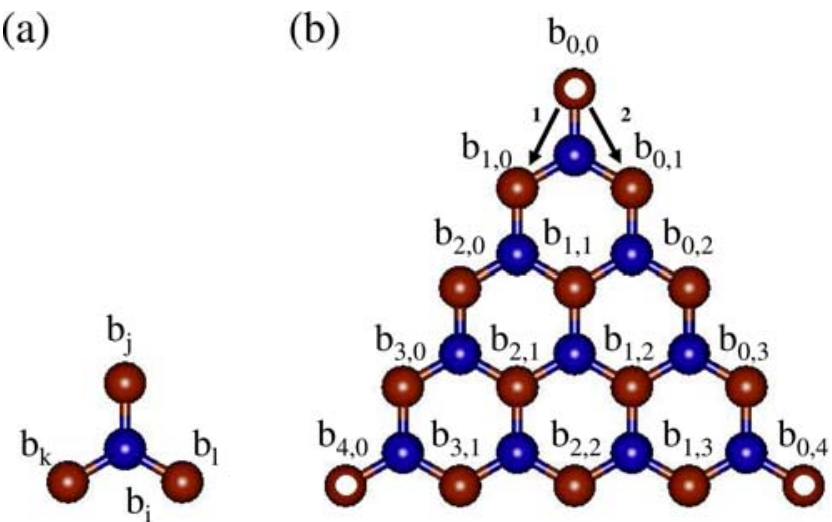

Figure 3 (a) An arbitrary $i$-th B-type site (blue circle) surrounded by three A-type sites, $j$-th, $k$-th, and $l$-th (red circles). (b) TGQD with $N_{\text {ed }}=3$ atoms on one edge. Above each A-type atom are corresponding coefficients. Open circles indicate auxiliary A-type atoms in the three corners, which will help to introduce three boundary conditions. For zero-energy states all coefficients can be expressed as superpositions of coefficients from the one edge, the left edge of atoms in our case. Reprinted from [1]. where $b_{i}, b_{j}, b_{k}, b_{l}$ are expansion coefficients. Using the zero-energy state condition, $H_{\mathrm{TB}} \Psi=0$, and projecting onto $\varphi_{z}^{i}$ we get

$$
\left\langle\varphi_{z}^{i}\left|H_{\mathrm{TB}}\right| \Psi\right\rangle=0 \cdot b_{i}+t \cdot b_{j}+t \cdot b_{k}+t \cdot b_{l}=0,
$$

where we use expressions $\left\langle\varphi_{z}^{i}\left|H_{\mathrm{TB}}\right| \varphi_{z}^{j}\right\rangle=\left\langle\varphi_{z}^{i}\left|H_{\mathrm{TB}}\right| \varphi_{z}^{k}\right\rangle=$ $\left\langle\varphi_{z}^{i}\left|H_{\mathrm{TB}}\right| \varphi_{z}^{l}\right\rangle=t$ and $\left\langle\varphi_{z}^{i}\left|H_{\mathrm{TB}}\right| \varphi_{z}^{i}\right\rangle=0$. Finally, Eq. (4) is written as

$$
b_{j}+b_{k}+b_{l}=0 \text {. }
$$

We will now apply the above analysis to a TGQD, as shown in Fig. 3(b), where the coefficients $b_{n, m}$ are now indexed according to their position vector $\boldsymbol{R}=n \boldsymbol{a}_{1}+m \boldsymbol{a}_{2}$. The structure has three auxiliary atoms attached with the coefficients $b_{0,0}, b_{0,4}, b_{4,0}$, which will later define appropriate boundary conditions. Starting from the top of the TGQD, Eq. (5) gives

$$
\begin{aligned}
& b_{0,1}=-\left(b_{0,0}+b_{1,0}\right), \\
& b_{1,1}=-\left(b_{1,0}+b_{2,0}\right) .
\end{aligned}
$$

The two equations above will help to determine the coefficients of the lower row of red atoms. By inspection, it is then possible to write a general compact form for coefficients $b_{n, m}$ :

$$
b_{n, m}=(-1)^{m} \sum_{k=0}^{m}\left(\begin{array}{l}
m \\
k
\end{array}\right) b_{n+k, 0} .
$$

Here, it is important to emphasize that the only unknowns are the $N_{\text {ed }}+2$ coefficients, $b_{n, 0}$ 's, from the left edge; the rest are expressed as their superpositions, as seen from Eq. (8). In addition, we must use the boundary conditions: the wave function has to vanish on three auxiliary atoms in each corner, see Fig. 3(b). This gives three boundary con- 
ditions, for TGQD from Fig. 3(b) $\left(b_{0,0}=b_{4,0}=b_{0,4}=0\right)$, or for arbitrary-size triangle $\left(b_{0,0}=b_{N_{\text {ed }}+1,0}=b_{0, N_{\text {ed }}+1}=0\right)$, reducing the number of independent coefficients to $N_{\text {ed }}-1$. The number of linearly independent coefficients corresponds to the maximum number of linearly independent vectors and determines the dimension of the degenerate zero-energy shell $N_{\text {deg }}=N_{\text {ed }}-1$, confirming previous numerical calculations with results shown in Fig. 2.

A similar analysis can be done for B-type atoms showing that they must all equal zero. Finally, a general form for the eigenvectors for zero-energy states in the triangle can be written as

$$
\Psi=\sum_{n=0}^{N_{\mathrm{ed}}+1} \sum_{m=0}^{N_{\mathrm{ed}}+1-n}\left[(-1)^{m} \sum_{k=0}^{m}\left(\begin{array}{l}
m \\
k
\end{array}\right) b_{n+k, 0}\right] \varphi_{n, m}^{\mathrm{A}} .
$$

In this expression only $N_{\text {ed }}-1$ coefficients corresponding to atoms from the one edge are independent. We can construct $N_{\text {ed }}-1$ linearly independent eigenvectors, which span the subspace of zero-energy states. Thus, the number of zero-energy states in the triangle is $N_{\text {deg }}=N_{\text {ed }}-1$. This can be also related to the imbalance between the number of atoms belonging to each sublattice, $N_{\mathrm{deg}}=N_{\mathrm{A}}-N_{\mathrm{B}}$.

\subsection{Zero-energy states in a magnetic field The} analysis of the zero-energy states can also be generalized to non-zero external magnetic fields [28]. In this case, the wave function coefficients given in the bracket in Eq. (9) become

$$
b_{n, m}(\varphi)=(-1)^{m} \sum_{k=0}^{m} \frac{1-\mathrm{e}^{2 \pi i\left(\begin{array}{l}
m \\
k
\end{array}\right) \frac{\varphi}{\phi_{0}}}}{1-\mathrm{e}^{2 \pi i \frac{\varphi}{\varphi_{0}}}} \mathrm{e}^{-i \phi_{n+k}} b_{n+k, 0},
$$

where $\varphi_{0}=\frac{h c}{e}$ is the magnetic flux quantum, $\varphi=B_{z} S_{0}$ is the magnetic flux threading one benzene ring, $S_{0}=3 \sqrt{3} a_{0}^{2} / 2$ is the benzene ring area with $a_{0}=1.42 \AA$, and $\phi_{n+k}$ represents the phase corresponding to the path on the right edge connecting sites $\{n+k, 0\}$ and $\{n, m\}$ [28]. Note that Eq. (10) reduces to Eq. (8) when $\varphi=0$. Interestingly, Eq. (10) shows that the zero energy states in triangular graphene quantum dots survive in external magnetic fields, the only effect is the Zeeman splitting. The effect is similar to the appearance of the $n=0$ Landau level in bulk graphene. When the cyclotron energy becomes comparable to the energy gap, the zero-energy shell and electron and hole states evolving toward the $n=0$ Landau level overlap energetically [28].

For TGQDs, the crossings of valence and conduction state at $E=0$ were investigated analytically and numerically. Figure 4 shows the energy gap as a function of $\varphi / \varphi_{0}$ for different $N_{\text {ed }}$ obtained by diagonalization of the tightbinding Hamiltonian. Strikingly, the first crossing always occurs at $\varphi / \varphi_{0}=1 /\left(N_{\text {ed }}+1\right)$ for all the values of $N_{\text {ed }}$. The crossing of valence and conduction states at $E=0$ opens

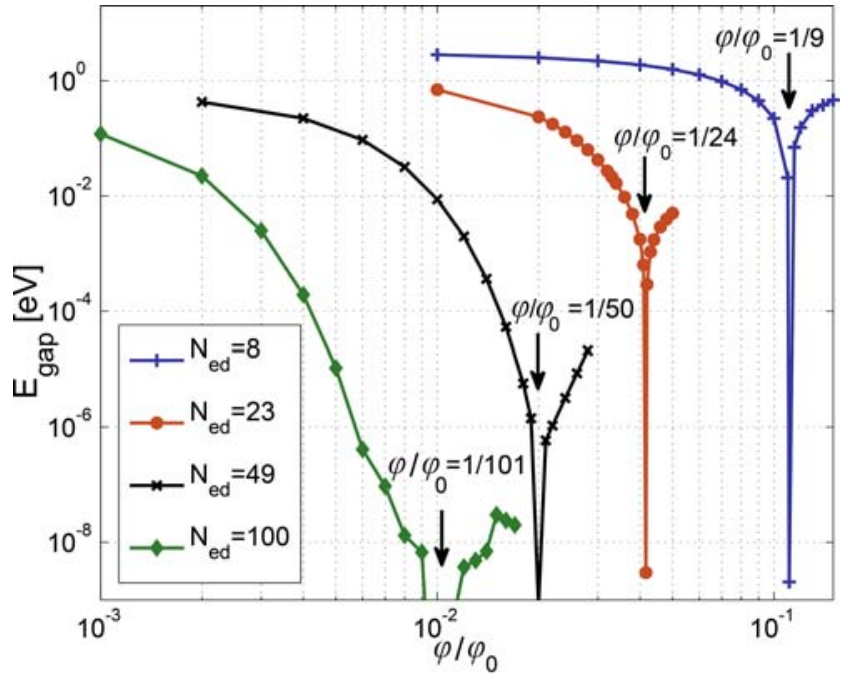

Figure 4 Energy gap between lowest conduction and highest valence states as a function of magnetic flux for different $N_{\text {ed }}$. First zero energy crossing occurs at $\varphi / \varphi_{0}=1 /\left(N_{\text {ed }}+1\right)$. Reprinted from [28].

the possibility to manipulate strongly correlated electronic systems of the degenerate zero-energy shell [28].

2.4 Bilayer triangular graphene quantum dots with zigzag edges In this subsection we investigate the effect of inter-layer coupling and an external perpendicular electric field on the zero-energy states of bilayer triangular quantum dots with zigzag edges (BTGQD) in AB Bernal stacking, shown in Fig. 5(a). The top-layer triangle (blue color) is slightly smaller in size than the bottom triangle so that there are no floating atoms. Regardless of the size, such construction has always an odd number of zero energy states. In order to study single particle properties, we diagonalize the tight-binding Hamiltonian given by

$$
H_{\mathrm{TB}}=\sum_{i j \sigma} t_{i j} c_{i \sigma}^{\dagger} c_{j \sigma}+\sum_{i \sigma} V_{i} c_{i \sigma}^{\dagger} c_{i \sigma}
$$

where the hopping parameters $t_{i j}$ include an inter-layer coupling between the top-A and bottom-B atoms given by $\varphi_{\mathrm{i}} t_{\perp}=-0.4 \mathrm{eV}$ in addition to the usual in-plane nearest neighbor tight-binding parameters fixed to $t=-2.8 \mathrm{eV}$. Under the external perpendicular electric-field, a potential difference between the upper quantum dot atoms $\left(V_{i}=-\Delta V / 2\right)$ and lower quantum dot atoms $\left(V_{i}=\Delta V / 2\right)$ is induced. In Fig. 5(b) and (c), the tight-binding spectrum around the Fermi level for a BTGQD consisting of 1195 atoms and $N_{\text {deg }}=43$ zero-energy states is investigated. For this structure, the edge sizes for the upper and lower layers are $N_{\text {ed }}=23$ and $N_{\text {ed }}=22$, respectively. When $\Delta V=0$ (Fig. 5(b)), the value of $t_{\perp}$ has no effect on the degenerate shell, as if the two layers were decoupled. When we turn on the external electric field (Fig. 5(c)), the degeneracy between the upper and lower-layer zero-energy states is lifted by $\Delta V=0.4 \mathrm{eV}$. An intriguing aspect of 

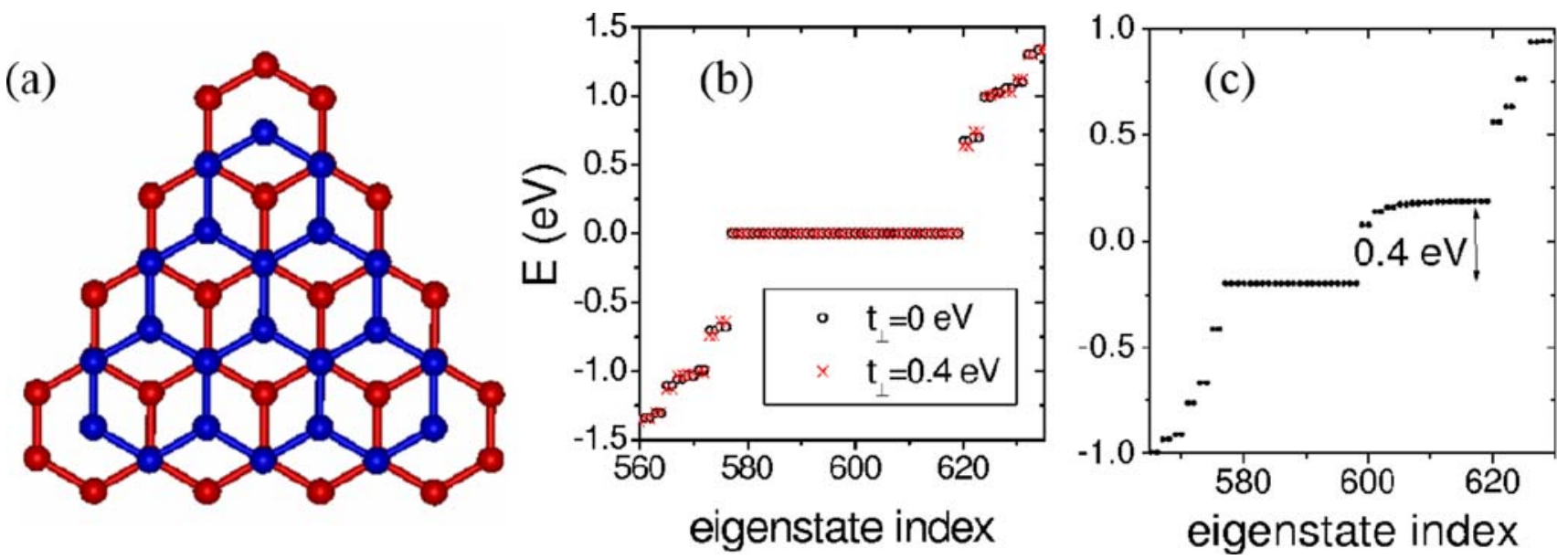

Figure 5 (a) A bilayer TGQD. (b) Tight-binding spectrum for a bilayer TGQD with 1195 atoms that has $N_{\text {deg }}=43$ zero-energy states. (c) Under external electric field, the degeneracy between the 21 top-layer zero-energy states and 22 bottom-layer zero-energy states is lifted. Reprinted from Ref. [24].

Fig. 5(c) is the absence of dispersion in the zero-energy states corresponding to the bottom layer. Indeed, the bottom-layer zero-energy states belong solely to the sublattice atoms that do not couple to the top-layer. On the other hand, top-layer zero-energy states do couple to the bottomlayer through $t_{\perp}$. In the following section, we will discuss how the magnetization of BTGQDs can be controlled by tuning the relative position of zero-energy states.

3 Voltage control of magnetic properties of TGQDs In this section, we describe the magnetic properties of TGQDs using a methodology that combines tightbinding, Hartree-Fock, and configuration interaction techniques $(\mathrm{TB}+\mathrm{HF}+\mathrm{CI})$ explained in this issue by Ozfidan et al. As discussed above, the broken sublattice symmetry in TGQDs gives rise to a shell of degenerate levels at the Fermi level. It is also expected to lead to magnetism according to Lieb's theorem on half-filled bipartite Hubbard model. However, under external voltage, the system is away from half-filling where long range interactions and other electron-electron scattering events may become important. We will discuss how the electronic and magnetic properties of TGQDs depend on the filling of the shell, how they can be controlled by electric field in bi-layer TGQDs and how they can be detected in Coulomb and spin blockade transport experiments.

\subsection{Filling factor dependence of the total spin} of TGQD In order to study the filling factor dependence of magnetic properties in the degenerate shell beyond a meanfield description, we first perform a Hartree-Fock calculation for the charged system of $N-N_{\text {deg }}$ electrons, with empty degenerate shell. As shown in Fig. 6 (black lines), a group of three states becomes separated from the zeroenergy shell by a small gap of $\sim 0.2 \mathrm{eV}$. These states are localized at the corners of the TGQD as shown in the inset. A comparison with density functional calculations within

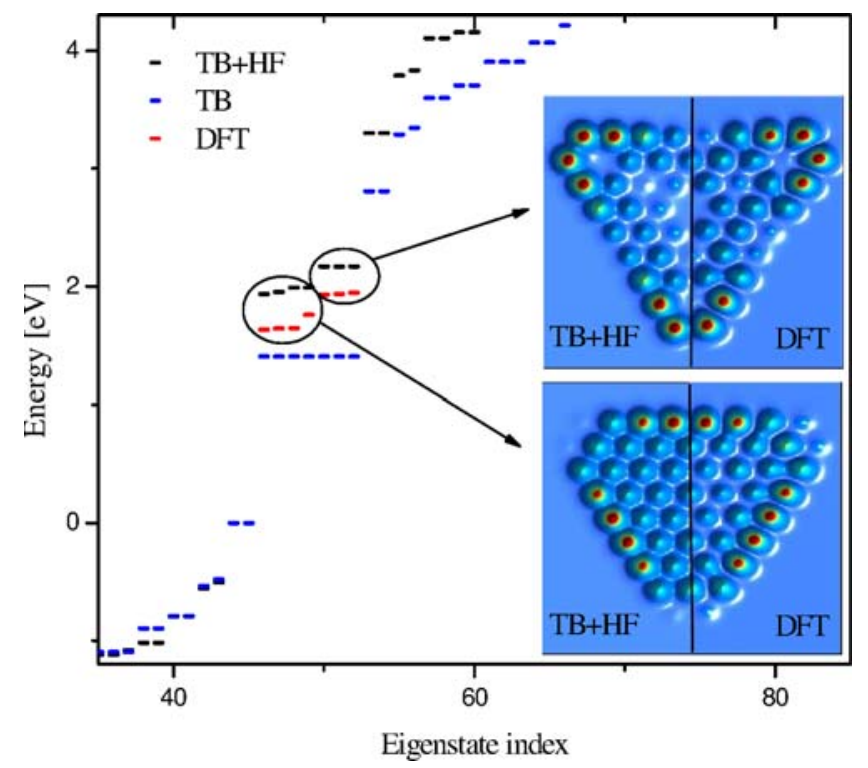

Figure 6 Single particle spectrum of a TGQD with 97 carbon atoms with empty zero-energy shell, obtained by tight-binding (TB, blue lines) and self-consistent Hartree-Fock (TB + HF, black lines) methods. The 7 zero-energy states near the Fermi level are compared to DFT results. The dielectric constant $\kappa$ is set to 6 . The inset compares the structure of corner and side states obtained using Hartree-Fock and DFT calculations. Reprinted from Ref. [21].

local density approximation is also provided in Fig. 6. The separation of corner states also occurs in density functional calculations, confirming the Hartree-Fock results. In the following, we will investigate the many-body effects within the degenerate shell by performing configuration interaction calculations as a function of number of electrons in the shell, using the HF states as a basis set.

Figure 7 shows the dependence of the low-energy many-body spectra obtained using TB + HF + CI method- 

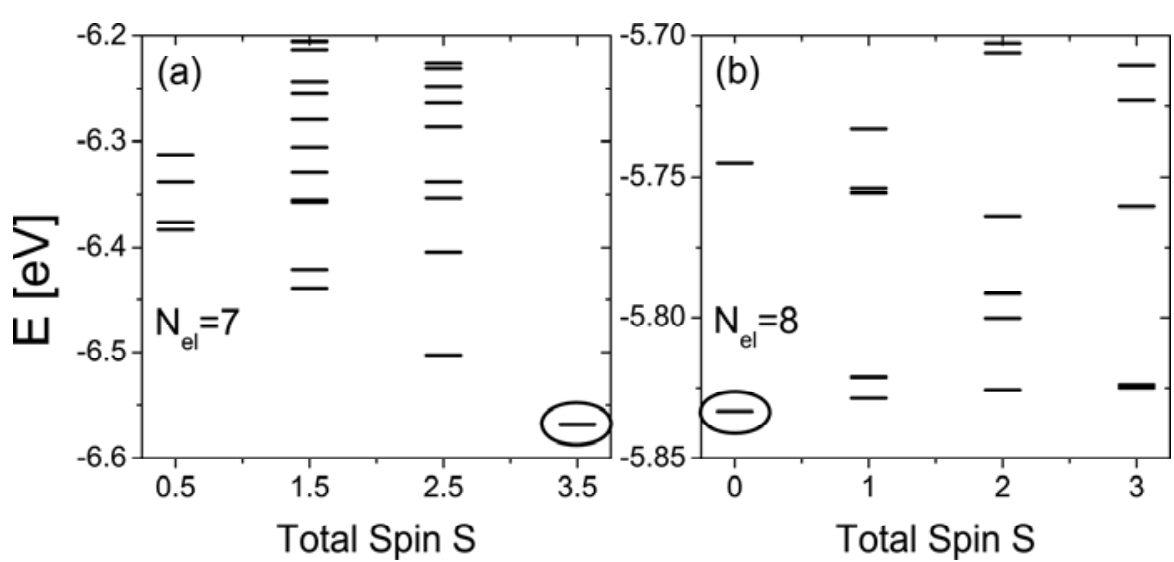

Figure 7 Many-body spectra for the different total spin $S$ for (a) charge neutral case $\left(N_{\mathrm{el}}=7\right)$ and (b) charged case $\left(N_{\mathrm{el}}=8\right)$. For $N_{\mathrm{el}}=7$, the ground state total spin is maximized, indicated by a circle. For $N_{\mathrm{el}}=8$ electrons the ground state is depolarized to $S=0$, and is almost degenerate with excited states with different total spin $S$. Reprinted from Ref. [27]. ology on the total spin $S$ for a TGQD with $N=97$ carbon atoms which exhibit $N_{\text {deg }}=7$ zero-energy states [21, 27]. Charge neutral (half-filled) case with $N_{\text {el }}=7$ electrons on the degenerate shell (Fig. 7(a)), and charged case, i.e., $N_{\text {el }}=8$ electrons (Fig. 7(b)) are considered. In agreement with previous calculations based on mean-field approaches [15-17], when the system is charge-neutral, i.e., $N_{\mathrm{el}}=7$, there is a finite magnetization with $S=3.5$, indicated by a circle. This many-body state corresponds to a single configuration where there is exactly one spin-down electron per degenerate state. Its total energy is well separated from the lower $S$ states which requires at least one flipped spin among seven initially spin-polarized electrons. When an extra electron is added through, for instance, an external gate, the spectrum changes drastically as seen in Fig. 7(b). In particular, the ground state is now depolarized with $S=0$, indicated by a circle. This new ground state is almost degenerate with states corresponding to the different total spin, which is a signature of strong electronic correlations. The size dependence of the spin depolarization was also studied in Ref. [27]. It was shown that for larger TGQDs with $N_{\text {deg }}>9$ a partial depolarization still occurs but at higher filling factors.

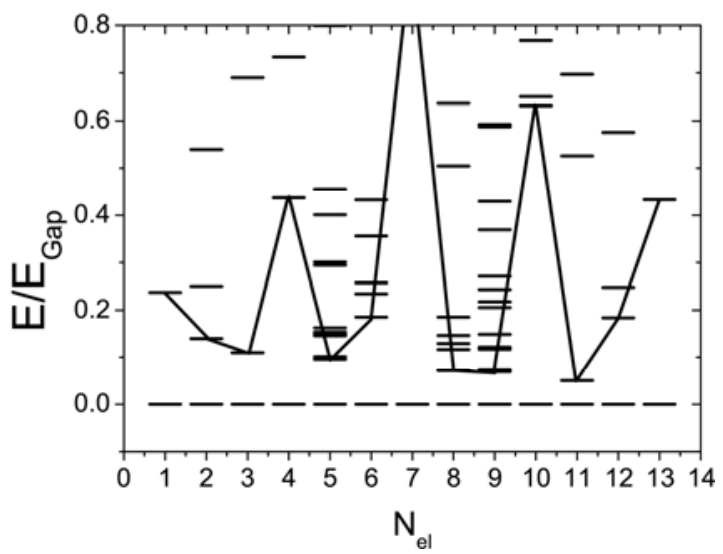

Figure 8 Many-body spectrum as a function of the number of electrons occupying the zero-energy states with degeneracy $N_{\text {deg }}=7$. The energies are renormalized by the energy gap corresponding to the half-filled shell, $N_{\mathrm{el}}=7$ electrons. Reprinted from Ref. [27].
Figure 8 shows the many-body spectrum for the $N_{\text {deg }}=7$ case, as a function of the electronic occupancy of the degenerate shell. The energies are renormalized by the energy gap corresponding to the half-filled shell, i.e., $N_{\text {el }}=7$ electrons. The solid line shows the evolution of the energy gap as a function of shell filling. For $N_{\mathrm{el}}=7$ (charge neutral case), as well as for $N_{\mathrm{el}}=7-3=4$ and $N_{\mathrm{el}}=7+3=10$, the energy gaps are found to be considerably higher than the other fillings. Moreover, the decrease of the energy gap around charge neutrality is accompanied by an increased density of states at the lower energy spectrum, indicating strong electronic correlations. These results show that the electronic and magnetic properties of the system can be modified drastically through applied voltage.

Figure 9(a) shows the spin phase diagram as a function of the number of electrons occupying the degenerate shell for the $N_{\mathrm{deg}}=7$ case. The total spin $S$ is maximized for

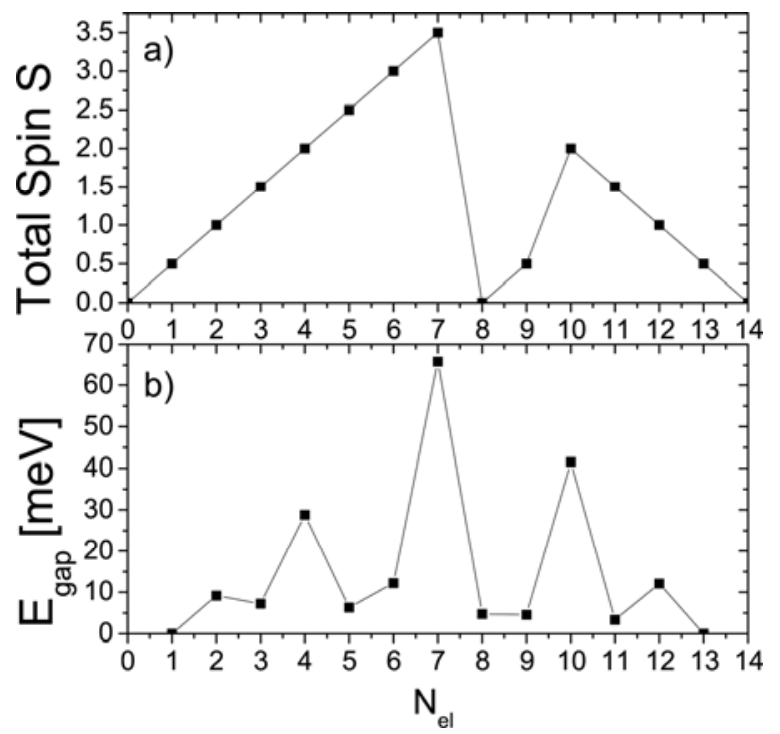

Figure 9 (a) Total spin as a function of the number of electrons occupying the degenerate shell for the same TGQD as in Fig. 8 and (b) corresponding to the energy excitation gaps. The magnitude of the energy gap at certain fillings is significantly reduced due to correlation effects. Reprinted from Ref. [27]. 

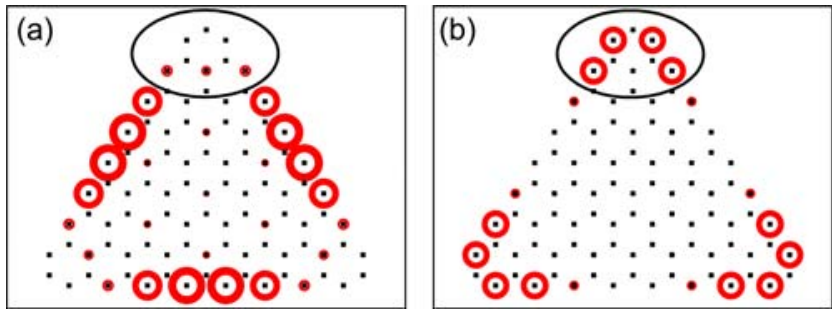

Figure $10 \mathrm{Spin}$ densities of the ground state for (a) $N_{\mathrm{el}}=4$ electrons and (b) $N_{\mathrm{el}}=10$ electrons that correspond to subtracting/adding three electrons from/to the charge-neutral system. The radius of circles is proportional to a value of spin density on a given atom. A long-range Coulomb interaction repels (a) holes and (b) electrons to three corners, forming a spin-polarized Wigner-like molecule. Reprinted from Ref. [27].

most electron numbers, except for $N_{\mathrm{el}}=8,9$ electrons. On the other hand, Fig. 9(b) reveals a strong oscillation of the energy gap as a function of shell filling as a result of a combined effect of correlations and system's geometry, indicating a competition between fully spin polarized TGQD that maximizes exchange energy and fully unpolarized system that maximizes the correlation energy. For $N_{\mathrm{el}}=8$ and $N_{\text {el }}=9$, correlation energy wins over the exchange energy resulting in a minimized ground state total spin, accompanied by a large density of states, shown in Fig. 8.

In order to investigate further the large excitation gaps for $N_{\text {el }}=4$ and $N_{\text {el }}=10$ electrons seen in Fig. 9(b), we plot the corresponding spin densities in Fig. 10. Here, longrange mean-field interactions dominate the physics. Thus, consistent with the triangular symmetry of the system, three spin polarized holes $\left(N_{\mathrm{el}}=7-3\right.$ electrons) and electrons $\left(N_{\mathrm{el}}=7+3\right.$ electrons) maximize their relative distance by occupying three consecutive corners. The formation of localized holes can be understood in terms of the Hartree-Fock orbitals of empty degenerate shell discussed in Fig. 6. Aided by exchange energy, first four electrons occupy the side states shown in Fig. 6, leaving behind three corner holes. On the other hand, when $N_{\text {el }}=7$ electrons are added to the shell, the HF quasiparticle energies are renormalized to a perfectly flat shell as in the TB model. Then, when three more electrons are added, they form a spin polarized Wigner-like molecule [40, 41], resulting from long-range interactions which dominate over the flat band kinetic energy and triangular geometry.

3.2 Coulomb and spin blockades in TGQD Magnetic properties of quantum dots can be probed using the Coulomb and spin blockade spectroscopy [42]. When the conductance through a quantum dot weakly coupled to leads is measured as a function of gate voltage, a series of peaks are obtained as new electrons are loaded from the gate. These peaks, called Coulomb blockade peaks, occur due to a combined effect of size quantization and Coulomb repulsion. The position and amplitude of these peaks give information about the density dependent electronic and magnetic properties of the quantum dot. The amplitude of the Coulomb blockade peak is given by the conductivity $G_{i}$ of the graphene quantum dot connected to leads via atom " $i$ " [43] as shown schematically in Fig. 11(a). The amplitude of the Coulomb blockade peak can be calculated using the transition probability $\left\langle\left.\left\langle N_{\mathrm{el}}+1, J^{\prime}, S^{\prime}\left|c_{i \sigma}^{\dagger}\right| N_{\mathrm{el}}, J, S\right\rangle\right|^{2}\right.$ from the many-body state $\left(N_{\mathrm{el}}, J, S\right)$ to the state $\left(N_{\mathrm{el}}+1, J^{\prime}, S^{\prime}\right)$ when an additional electron is added to the site " $i$ " of the graphene quantum dot from the lead. Figure 11(b) shows the conductivity for a TGQD with $N_{\text {deg }}=7$ degenerate zero-energy states. Although, one would expect a total of fourteen peaks reflecting the degeneracy of the zero-energy shell, only eleven peaks are observed in Fig. 11(b). In fact, due to a phenomenon called spin blockade, some of the peaks vanish. For instance, the transitions from $\left(N_{\mathrm{el}}=7, S=7 / 2\right)$ states to $\left(N_{\mathrm{el}}=8, S=0\right)$ states are spin blocked since it is not possible to change the spin of the system by $\Delta S=-7 / 2$ by adding one electron
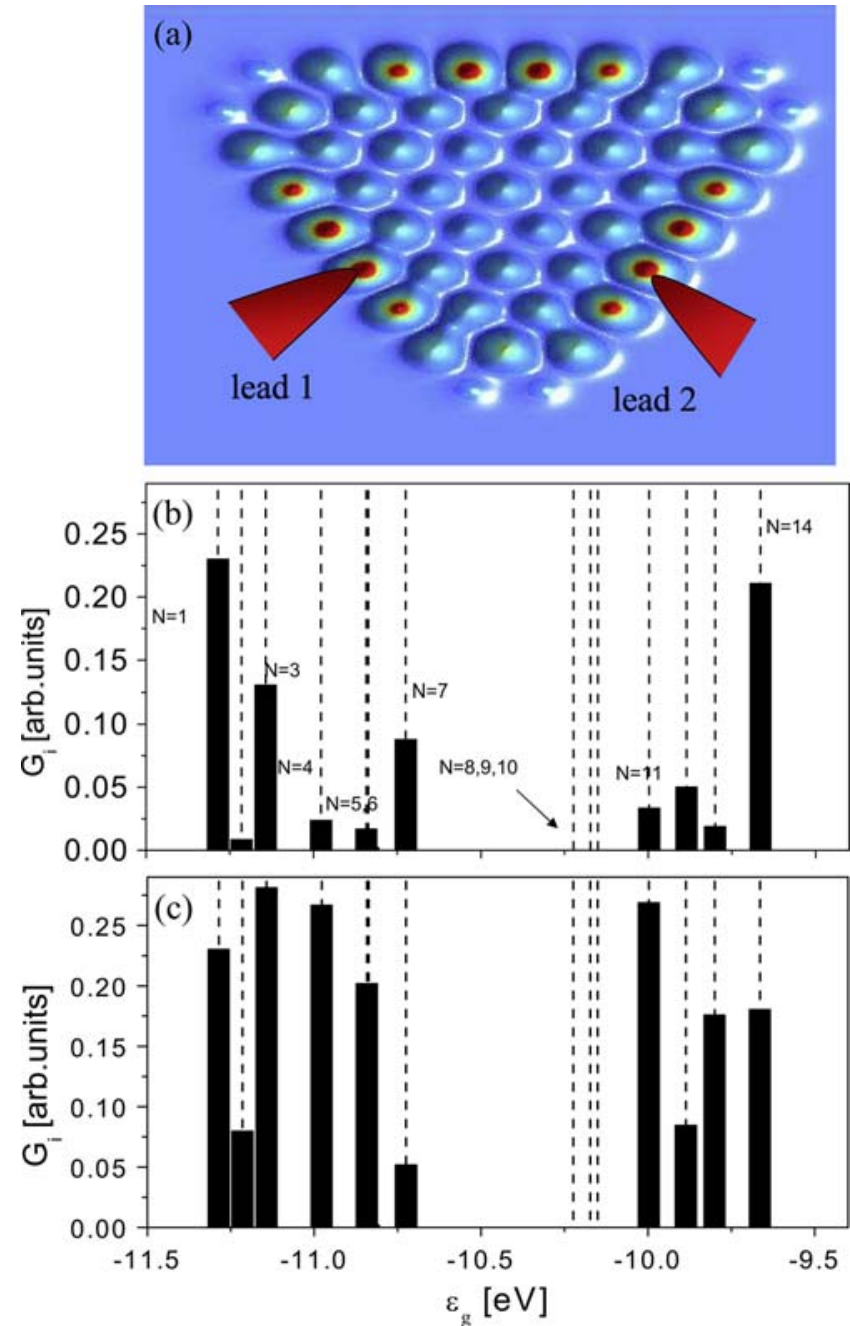

Figure 11 (a) Schematic representation of TGQD connected to the leads through a side site. (b) Conductivity as a function of applied gate voltage, $\varepsilon_{\mathrm{g}}$. (c) Same as (b) but without the site dependence of the incoming electron. Reprinted from Ref. [21]. 
with $S=1 / 2$. Similarly, transitions from $\left(N_{\mathrm{el}}=9, S=1 / 2\right)$ states to $\left(N_{\mathrm{el}}=10, S=4 / 2\right)$ states are spin blocked.

In order to investigate the nature of the oscillations of the conductivity amplitudes as a function of gate voltage, in Fig. 11(c) we plot the conductivity assuming that the weight of the site " $i$ " does not depend on the zero-energy orbital. As a result, the weights of the peaks considerably change, indicating that, in addition to the stronglycorrelated nature of the states $\left|N_{\text {el }}, S\right\rangle$, the specific choice of the site where the lead is attached also plays an important role. These results show how to detect the spin depolarization in transport experiments. Ultimately, we show here that one can design a strongly correlated electron system in carbon-based material whose magnetic properties can be controlled by applied gate voltage.

4 Bilayer triangular graphene quantum dots with zigzag edges In Section 2.4 we showed that in a bilayer triangular quantum dot, the zero-energy states are not affected by the coupling between the two layers. Hence, we have two sets of zero-energy states originating from each layer. Moreover, we have seen that it is possible to control the relative energies of two sets of zero-energy states by applying an external electric field. In this section we will discuss the magnetic properties of edges and show that the ability of controlling the relative position of the energy of the bilayer graphene quantum dot gives an interesting opportunity to control the charge and spin of the zero-energy states. Most calculations in this section were performed using the mean-field extended Hubbard approximation. In all calculations the on-site Hubbard term $U$ is taken to be $2.75 \mathrm{eV}$, screened by a factor of $\sim 6$ from the bare Coulomb potential [24].

Figure 12 shows the spin density isosurfaces for zero electric field (left-hand side) and finite electric field (righthand side), as obtained from configuration interaction calculations. In the absence of the external electric field, both layers have a finite magnetic moment, differing by one spin due to the size difference of the two triangles. The inter-layer magnetic coupling is ferromagnetic, in agreement with Lieb's theorem which applies for Bernal stacking. When a sufficiently high electric field is applied, electrons from the lower layer reduce their energy by transferring to the top layer, occupying all the available spin-up and down zero-energy states, leaving behind one single spin.

In Fig. 13, we present a detailed phase diagram that leads to the transition described in Fig. 12. Figure 13(a) shows the energies for different total spin projection $S_{z}$ with respect to the energy of the ferromagnetic configuration, $S_{z}=9 / 2$. At $\Delta V=0$, all nine electrons occupying the degenerate shell have their spins aligned, in agreement with Lieb's theorem. As $\Delta V$ is increased, the electrons occupying the bottom layer zero-energy states are pushed towards the top layer. At a critical value of the electric potential, $\Delta V_{\mathrm{c}}=0.55 \mathrm{eV}$ the electrons gain enough energy to overcome the energy for flipping their spin and the charge transfer becomes possible. As a result, all the top layer
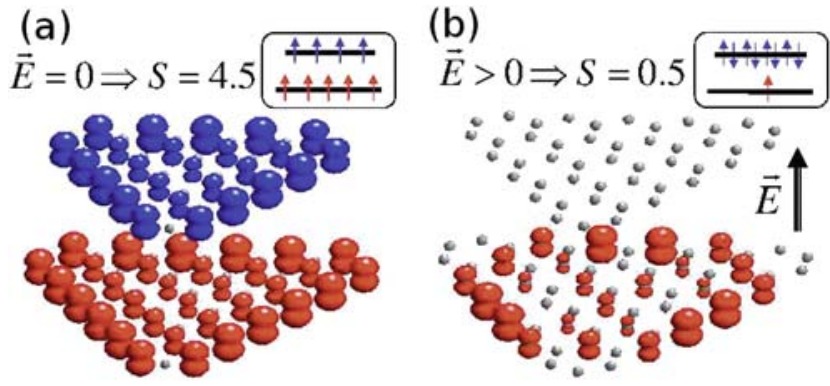

Figure 12 Isosurface plot of the spin density $\rho_{\uparrow}-\rho_{\downarrow}$ of a bilayer triangular graphene quantum dot with zigzag edges, (a) in the absence and (b) in the presence of a perpendicular electric field obtained from configuration interaction calculations. Reprinted from Ref. [24].

(layer 2) zero-energy states are doubly occupied and the magnetization of the system decreases abruptly, leaving exactly one single spin in the bottom layer (layer 1). We note that one can also isolate a single hole spin in the bottom layer by applying a reverse electric field, thus pushing the electrons from the top layer to the bottom layer, occupying all states except one. It is thus possible to isolate a single electron or hole spin in a neutral bilayer graphene quantum dot isolated from metallic leads by applying an external electric field.

In order to investigate the size dependence of the ferromagnetic (FM, where the ground state spin is given by $S_{z}^{\max }=N_{\text {deg }} / 2$ ) to antiferromagnetic (AFM, where the ground state spin is $S_{z}^{\min }=1 / 2$ ) transition for bilayer TGQDs described above, in Fig. 14(a) we show the FMAFM energy difference in the mean-field extended Hubbard approximation as a function of electric potential difference $\Delta V$, for different quantum dot sizes up to 1507 atoms. In the absence of external electric field, the FM-AFM gap increases with the size of the system $N$. The
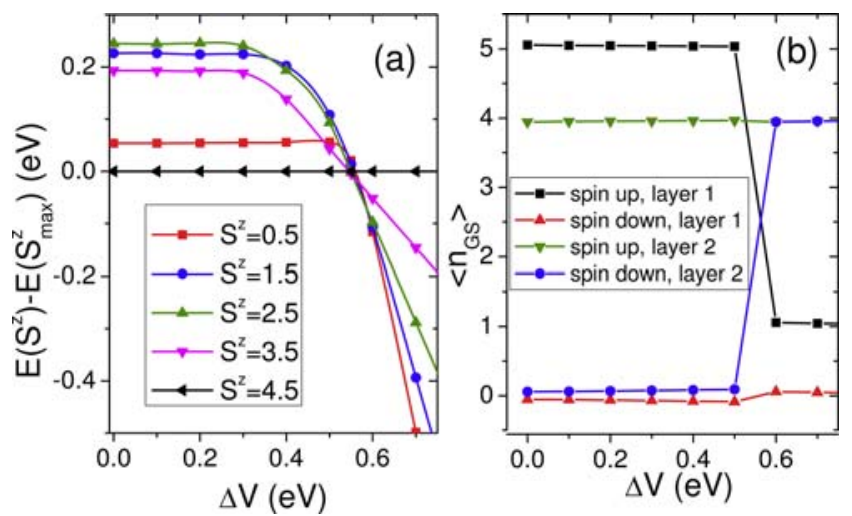

Figure 13 Mean-field Hubbard results for a bilayer graphene quantum dot with 107 atoms and 9 zero-energy states. (a) Energies of lowest energy states with different total spin projection $S_{z}$ as a function of potential difference $\Delta V_{\mathrm{c}}$ between the lower layer (layer 1) and upper layer (layer 2), with respect to the ferromagnetic configuration $S_{z}^{\max }=4.5$. (b) Ground state spin population for a given layer and spin. Reprinted from Ref. [24]. 

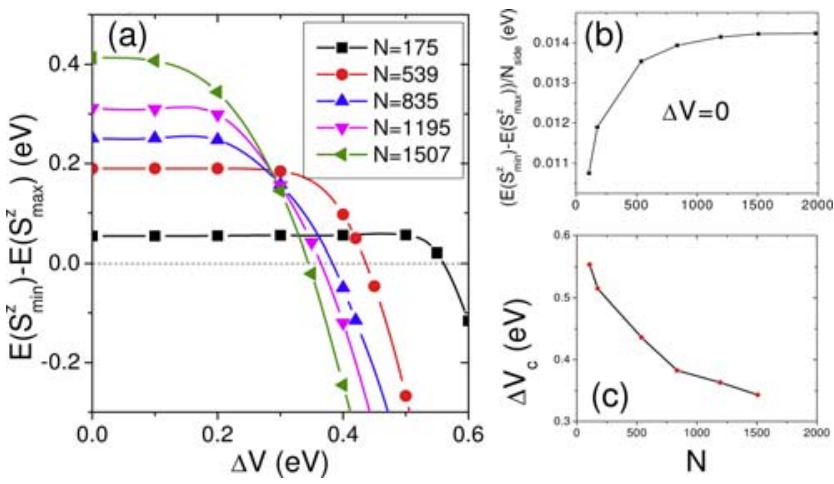

Figure 14 (a) FM-AFM energy difference as a function of potential difference $\Delta V$, for different quantum dot sizes up to $N=1507$ atoms. (b) For $\Delta V=0$, the FM-AFM energy gap per number of side atoms $N_{\text {side }}$ approaches $14.3 \mathrm{meV}$. (c) Critical value $\Delta V_{\mathrm{c}}$ where the FM-AFM transition occurs as a function of number of atoms $N$. Reprinted from Ref. [24].

energy gap per unit length of the triangle with linear size $N_{\text {side }}$ approaches a constant value of $14.3 \mathrm{meV}$ as shown in Fig. 14(b). However, the FM-AFM transition voltage $V_{\mathrm{c}}$ decreases with the system size as can be seen from Fig. 14(c). For the largest system size studied, $N=1507$, we obtain $\Delta V_{\mathrm{c}}=0.345 \mathrm{eV}$, which corresponds to an electrical field of $\sim 1 \mathrm{~V} / \mathrm{nm}$.

We note that, it is also possible to use an in-plane electric-field to tune the magnetization of a single layer TGQD [26].

5 Conclusion In summary, single- and bi-layer triangular graphene quantum dots with zigzag edges have unique electronic and magnetic properties arising from a broken sublattice symmetry. In particular, they exhibit a degenerate shell of spin polarized zero-energy states with degeneracy proportional to the linear size of the structure. The spin polarization can be tuned by an external voltage through electronic doping in single TGQDs. In bilayer TGQDs, the degeneracy of the upper and lower layer zeroenergy shells can be lifted by the external electric field allowing the control of relative filling of the shells and the reduction of the magnetization of the quantum dot down to a single localized spin.

Acknowledgements ADG acknowledges support from Bilim Akademisi - The Science Academy, Turkey, under the BAGEP program, and from Turkiye Bilimsel ve Teknolojik Arastirma Kurumu (TUBITAK), Turkey, under the BIDEP program. PP thanks for partial financial support from the sources granted for science development in the year 2013-2016, Grant no. IP2012 007372. PH acknowledges support from NSERC, University of Ottawa and NRC Canada.

\section{References}

[1] A. D. Güçlü, P. Potasz, M. Korkusinski, and P. Hawrylak, Graphene Quantum Dots (Springer, Berlin, Heidelberg, 2014).
[2] B. Trauzettel, D. V. Bulaev, D. Loss, and G. Burkard, Nature 3, 192 (2007).

[3] C. Stampfer, J. Güttinger, F. Molitor, D. Graf, T. Ihn, and K. Ensslin, Appl. Phys. Lett. 92, 012102 (2008).

[4] S. Schnez, K. Ensslin, M. Sigrist, and T. Ihn, Phys. Rev. B 78, 195427 (2008)

[5] M. Wimmer, A. R. Akhmerov, and F. Guinea, Phys. Rev. B 82, 045409 (2010).

[6] T. Ihn, J. Güttinger, F. Molitor, S. Schnez, E. Schurtenberger, A. Jacobsen, S. Hellmüller, T. Frey, S. Dröscher, C. Stampfer, and K. Ensslin, Mater. Today 44, 20 (2010).

[7] M. L. Mueller, X. Yan, J. A. McGuire, and L. Li, Nano Lett. 10, 2679 (2010).

[8] M. Grujic, M. Zarenia, A. Chaves, M. Tadic, G. A. Farias, and F. M. Peeters, Phys. Rev. B 84, 205441 (2011).

[9] S. K. Hämäläinen, Z. Sun, M. P. Boneschanscher, A. Uppstu, M. Ijäs, A. Harju, D. Vanmaekelbergh, and P. Liljeroth, Phys. Rev. Lett. 107, 236803 (2011).

[10] W. Sheng, M. Korkusinski, A. D. Güçlü, M. Zielinski, P. Potasz, E. S. Kadantsev, O. Voznyy, and P. Hawrylak, Frontiers Phys. 7, 328 (2012).

[11] D. Subramaniam, F. Libisch, Y. Li, C. Pauly, V. Geringer, R. Reiter, T. Mashoff, M. Liebmann, J. Burgdörfer, C. Busse, T. Michely, R. Mazzarello, M. Pratzer, and M. Morgenstern, Phys. Rev. Lett. 108, 046801 (2012).

[12] M. Olle, G. Ceballos, D. Serrate, and P. Gambardella, Nano Lett. 12, 4431 (2012)

[13] I. Ozfidan, M. Korkusinski, A. D. Güçlü, J. McGuire, and P. Hawrylak, Phys. Rev. B 89, 085310 (2014).

[14] T. Yamamoto, T. Noguchi, and K.Watanabe, Phys. Rev. B 74, 121409 (2006)

[15] M. Ezawa, Phys. Rev. B 76, 245415 (2007).

[16] J. Fernandez-Rossier and J. J. Palacios, Phys. Rev. Lett. 99, 177204 (2007)

[17] W. L. Wang, S. Meng, and E. Kaxiras, Nano Lett. 8, 241 (2008).

[18] J. Akola, H. P. Heiskanen, and M. Manninen, Phys. Rev. B 77, 193410 (2008).

[19] M. Manninen, H. P. Heiskanen, and J. Akola, Eur. Phys. J. D 52, 143-146 (2009).

[20] W. L. Wang, O. V. Yazyev, S. Meng, and E. Kaxiras, Phys. Rev. Lett. 102, 157201 (2009).

[21] A. D. Güçlü, P. Potasz, O. Voznyy, M. Korkusinski, and P. Hawrylak, Phys. Rev. Lett. 103, 246805 (2009).

[22] A. V. Rozhkov and F. Nori, Phys. Rev. B 81, 155401 (2010).

[23] P. Potasz, A. D. Güçlü, and P. Hawrylak, Phys. Rev. B 81, 033403 (2010)

[24] A. D. Güçlü, P. Potasz, and P. Hawrylak, Phys. Rev. B 84, 035425 (2011)

[25] M. Zarenia, A. Chaves, G. A. Farias, and F. M. Peeters, Phys. Rev. B 84, 245403 (2011).

[26] W.-L. Ma and S.-S. Li, Phys. Rev. B 86, 045449 (2012)

[27] P. Potasz, A. D. Güçlü, A. Wójs, and P. Hawrylak, Phys. Rev. B 85, 075431 (2012).

[28] A. D. Güçlü, P. Potasz, and P. Hawrylak, Phys. Rev. B 88, 155429 (2013).

[29] K. Szalowski, Physica E 52, 46 (2013).

[30] W. Sheng, K. Luo, and A. Zhou, Phys. Rev. B 90, 085406 (2014). 
[31] E. H. Lieb, Phys. Rev. Lett. 62, 1201 (1989).

[32] Y. Ma, P. O. Lehtinen, A. S. Foster, and R. M. Nieminen, New J. Phys. 6, 68 (2004).

[33] H. Sevincli, M. Topsakal, E. Durgun, and S. Ciraci, Phys. Rev. B 77, 195434 (2008).

[34] A. D. Güçlü and N. Bulut, Phys. Rev. B 91, 125403 (2015).

[35] K. Wakabayashi, M. Sigrist, and M. Fujita, J. Phys. Soc. Jpn. 67, 2089 (1998).

[36] Y.-W. Son, M. L. Cohen, and S. G. Louie, Nature 444, 347 (2006).

[37] L. Yang, M. L. Cohen, and S. G. Louie, Phys. Rev. Lett. 101, 186401 (2008).
[38] O. V. Yazyev, R. B. Capaz, and S. G. Louie, Phys. Rev. B 84, 115406 (2011).

[39] P. R. Wallace, Phys. Rev. 71, 622 (1947).

[40] B. Wunsch T. Stauber, and F. Guinea, Phys. Rev. B 77, 035316 (2008).

[41] I. Romanovsky, Y. Yannouleas, and U. Landman, Phys. Rev. B 79, 075311 (2009).

[42] M. Ciorga, A. S. Sachrajda, P. Hawrylak, C. Gould, P. Zawadzki, S. Jullian, Y. Feng, and Z. Wasilewski, Phys. Rev. B 61, R16315 (2000).

[43] A. D. Güçlü, Q. F. Sun, H. Guo, and R. Harris, Phys. Rev. B 66, 195327 (2002). 